had been vaccinated previously with CVI-78 and $73 \%$ of those vaccinated previously with standard calf lymph. All children in both groups had a vesicular reaction either after the first or the second vaccination. Likewise, all children had a positive $\mathrm{HI}$ titer On the other hand, only $65 \%$ of those children initially vaccinated with CVI-78 vaccine had positive neutralizing antibodies after revaccination, whereas all children who received standard calf lymph both times had positive neutralizing antibodies. Nineteen percent of the CVI-78 group had no HI or neutralizing antibodies after primary vaccination. All of these children responded as primary vaccinees on challenge with standard calf lymph. The number of vaccinations in this trial is insufficient to determine to what degree vaccination with CVI-78 will reduce the incidence of complications associated with smallpox vaccine.

\section{REFERENCES AND NOTES}

Cutchins, E., Warren, J., and Jones, W. P.: The antibody response to smallpox vaccination as measured by a tissue culture plaque method. J. Immunol., 85 . $275-283(1960)$.

2. Hierholzer, J. C., Suggs, M. T., and Hall, E. C.: Standardized viral hemagglutination and inhibition tests. Il. Description and statistical evaluation. Appl. Microbiol., 18: 824-833 (1969).

3. Millar, J. D., Roberto. R. R., Wulff, H., Wenner, H. A., and Henderson, D. A.: Smallpox vaccination by intradermal jet injection. I. Introduction; background and results of pilot studies. Bull. World. Health Org., 4I: 749-760 (1969).

Copyright $@ 1975$ International Pediatric Research Foundation, Inc.
4. Neff, J M Millar, J, D., Roberto, R. R., and Wulff, H.: Smallpox vaccination by intradermal jet injection. III. Evaluation in a well vaccinated population. Bull. World. Health Org., 41: 771-778 (1969).

5. Roberto R. R. Wulff, H., and Millar, J. D.: Smallpox vaccination by intradermal jet injection. II. Cutaneous and serological responses to primary intradermal jet injection. World. Health Org., 41: 761-769 (1969). vaccination in children. Bull Wor

6. Wesley, R. B., Speers, W. C., Neff, J. M., Ruben, F. L., and Lourie, B. Evaluation of two kinds of smallpox vaccine: CVI-78 and calf lymph vaccine. 1. Clinical and serologic response to primary vaccination. Pediat. Res., 9 $624-628(1975)$.

7. Kindly provided as Dryvax, lot. no. 281601, by Wyeth Laboratories, Inc., Marietta, Pa.

8. Six children in the $\mathrm{CVI}-78 / \mathrm{CL}$ group and three children in the $\mathrm{CL} / \mathrm{CL}$ group were not available for serology at the 28 -day postvaccination follow-up.

9. Dr. W C. Speers was formerly a student at The Johns Hopkins University School . University of Colorado Medical Center. of Medicine. Present addres

0. Mr. R. B. Wesley was formerly a student at The Johns Hopkins University School of Medicine, Present address: USAF Hospital, Kirkland, New Mexico. 1. Dr. J. M. Neff is Associate Professor of Pediatrics, The Johns Hopkins University School of Medicine, Baltimore, Maryland.

12. Dr. J. Goldstein was formerly Epidemic Intelligence Service Officer, Center for Disease Control, United States Public Health Service, Atlanta, Georgia.

13. Dr. B Lourie was formerly Chief, Vesicular Disease Labs, Virology Section. Center for Disease Control, United States Public Health Service. Atlanta, Georgia.

14. Request for reprints should be addressed to: J. M. Neff, M.D.. The Johns Hopkins University School of Medicine, 725 N. Wolfe Street, Baltimore, Md. 21205 (USA).

15. Accepted for publication April 2. 1975.

\title{
Phenylalanine Hydroxylase Activity in Liver Biopsies from Hyperphenylalaninemia Heterozygotes: Deviation from Proportionality with Gene Dosage
}

\author{
SEYMOUR KAUFMAN AND EDWARD E. MAX \\ Laboratory of Neurochemistry. National Institute of Mental Health, Bethesda, Maryland, USA
}

ELLEN S. KANG

University of Tennessee, Department of Pediatrics, Memphis, Tennessee, USA

\section{Extract}

Liver biopsy samples from the patients with hyperphenylalaninemia have an average of $5 \%$ of the normal hydroxylase activity. The parents of the patients have between $7.3 \%$ (excluding the value for one parent) and $10 \%$ of the normal hepatic hydroxylase activity. An explanation for these findings involves negative interallelic complementation, which involves protein-protein interaction between subunits in a multimeric enzyme. In support of this model is the evidence that rat liver phenylalanine hydroxylase is a multimeric protein composed of two electrophoretically distinguishable subunits.

\section{Speculation}

The finding that parents of patients with hyperphenylalaninemia have an average of $10 \%$ of the normal level of hepatic phenylalanine hydroxylase, a multimeric enzyme, can be explained on the assumption that the liver tissue of heterozygotes has an excess of enzyme molecules that contain at least one mutant subunit.

Widespread screening of neonates for phenylketonuria (PKU) has in recent years brought to light a class of patients with an apparently milder defect in phenylalanine metabolism than is seen in classic PKU. These patients are characterized by serum phenylalanine concentrations which are well below the concentrations in PKU serum, but still severalfold higher than normal (8). The condition has therefore been called hyperphenylalaninemia. The patients can be distinguished from PKU heterozygotes, and their pedigrees suggest autosomal recessive genetics (15).

In this laboratory, a series of liver biopsies from several hyperphenylalaninemic patients was shown by direct enzyme assay 
to have about $5 \%$ of the normal phenylalanine hydroxylase activity of human liver $(9,11)$. As part of that study, the hydroxylase activity of the parents of three hyperphenylalaninemic children was also assayed with the identical technique, but the results were not reported (except for one set of parents, see Reference 9) because no conclusive interpretation could be made at that time. Although our interpretations must still be tentative. we would now like to present these data, since absolutely conclusive interpretation based on further experiments may not be forthcoming in the immediate future because of the unavailability of human liver tissue for assay.

\section{METHODS AND MATERIALS}

The assay for phenylalanine hydroxylase was the same as that used previously $(9-11)$. It is based on incubation of liver extracts with $\mathrm{L}-\left[{ }^{14} \mathrm{C}\right]$ phenylalanine, a tetrahydropterin (2-amino-4hydroxy-6,7-dimethyl tetrahydropterin), a tetrahydropterinregenerating system (dihydropteridine reductase plus reduced pyridine nucleotide), and excess catalase. The radioactivity in the $\left[{ }^{14} \mathrm{C}\right]$ tyrosine that is formed is determined after separation of the tyrosine from the phenylalanine by paper chromatography. All reported hydroxylase-specific activities represent values that were repeated in several independent assays. They were all carried out under conditions in which the hydroxylase activity is proportional to protein concentration and time of incubation. In addition, during the course of $\mathrm{K}_{\mathrm{m}}$ determinations $(4,9,11)$, most of the samples were assayed for hydroxylase activity at several different concentrations of either phenylalanine or pterin cofactor. Under all assity conditions, the relative hydroxylase activities were similar to those reported in Table 1. All materials were from sources that have been described previously $(9-11)$.

\section{RESULTS}

The results summarized in Table I show that liver biopsy samples from the patients with hyperphenylalaninemia have an average of 5\% of the normal hydroxylase activity. Although most of the control liver samples were from patients with liver disease. the last sample was from an accident victim: there is no indication this last value differs from the others.

For the parents, the values show a considerable scatter, raising the possibility that they do not constitute a homogeneous group. In particular, the relatively high hydroxylase activity of father Ke

Table 1. Liver phenylalanine hydroxylase activity

\begin{tabular}{lc}
\hline \multicolumn{1}{c}{ Subjects } & $\begin{array}{c}\text { Tyrosine, } \mu \mathrm{mol} / \mathrm{g} \\
\text { protein } / \mathrm{hr}\end{array}$ \\
\hline Hyperphenylalaninemia patients & \\
JKe & 4.7 \\
SP & 3.8 \\
$P G$ & 2.7 \\
Parents of hyperphenylatlanimenial patients & \\
Father Ke & 23.0 \\
Mother Ke & 7.2 \\
Father $P$ & 4.4 \\
Mother $P$ & 7.7 \\
Father $G$ & 5.8 \\
Mother $G$ & 11.4 \\
Normal controls & \\
1. Extra-hepatic block & 96 \\
2. Choledocholithiasis & 59 \\
3. Cholelithiasis & 71 \\
4. Biliary atresiat & 49 \\
5. Biliary atresia & 9.5 \\
6. Head injury: & 83
\end{tabular}

${ }^{1}$ This value has been published previously (4). The liver sample (from an accident victim) wals removed and frozen immediately after death. We are grateful to Dr. E. LaBrosse for obtaining this liver sample.
(31\% of the average control value) suggests that his genotype may be diflerent from that of the other parents (in which case, the genotype of $J K e$ would also be different from that of the other patients). It should be noted that the possibility that father $K e$ is a normal subject is unlikely, since we have found previously that both his fasting plasma phenylalanine levels and his phenylalanine levels $4 \mathrm{hr}$ after an oral phenylalanine load were elevaled compared with values for normal control subjects (9). All of the other parents also showed signs of abnormal phenylalanine metabolism (either elevated plasma phenylalanine-to-tyrosine ratios or abnormally high phenylalanine levels after oral phenylalanine loading (9)). Excluding father $K e$ from the group, the parents have an average of $7.3 \%$ of the normal hepatic hydroxylase activity; including him, the average value is $10 \%$ of the normal. This low value for the parents is of considerable interest, for although there have been other examples of heterozygotes with less than the expected $50 \%$ of normal activity, such as in cystathionine synthase deficiency in which the heterozygotes have about $34 \%$ of the normal level of the affected enzyme $(2,6,14)$, and adenine phosphoribosyl transferase deficiency in which the heterozygotes have between 21 and $37 \%$ of the normal activity (13), in most other cases where enzyme activities have been assayed in individuals heterozygous for enzyme deficiencies, proportionality with gene dosage has been observed (7). Therefore, if the hyperphenylalaninemia patients synthesize a hydroxylase variant with $5 \%$ of the normal activity, one might expect the parents (presumably heterozygotes) to show about $50 \%$ of the normal activity. Unfortunately, it is possible that the low activities that we have observed in the heterozygotes could be related to the biopsy method: percutaneous needle biopsies were used for the parents of the hyperphenylalaninemia children, whereas for the children themselves, and for all normal controlsubjects, open biopsies were used. We have made one attempt to assess the possible variation in hydroxylase determinations which might result from the difference in biopsy methods: the hydroxylase was assayed in both needle and open biopsies taken from a single dog, and the results were almost identical. Inaccessibility of tissue precludes a direct experiment to determine whether the same would in fact be true for human liver, but it seems unlikely that this differential biopsy technique could completely account for the low hydroxylase activity of the parents (16).

In a single mixing experiment, the hydroxylase activities of extracts from a control liver sample and a hyperphenylalaninemia liver sample were shown to be additive. This result suggests that the deficiency in hydroxylase activity observed in hyperphenylataninemia is not calused by the presence of inhibitors.

\section{DISCUSSION}

Apart from the possibilities of biopsy bias and en $\angle y m e$ inhibitors, both of which seem remote, two other interpretations may explain the deviation from proportionality of activity with gene dosage in the heterozygotes.

1. Regulator gene mutations have been the basis of models proposed in other cases of enzyme deficiencies in which heterozygote activity levels differed from expected values. Against this explanation for hyperphenylalianinemia heterozygotes is the evidence that phenylalanine hydroxylase in livers from hyperphenylalaninemia patients is distinguishable from the normal enzyme in the following properties $(4):(a)$ it has a lower apparent $\mathrm{K}_{\mathrm{m}}$ value for phenylalanine in the presence of 6.7-dimethyltetrahydropterin; $(b)$ its activity is stimulated less by lysolecithin in the presence of the natural cofactor, tetrahydrobiopterin: and (c) it appears to be somewhat more labile to heat inactivation. These results suggest that the low hydroxylase activity in hyperphenylalaninemic patients is caused by a mutation in the gene coding for the structure of the hydroxylase, rather than in a gene that regulates the rate of synthesis of the normal enzyme.

2. An alternative explanation would involve negative interallelic complementation. a phenomenon for which there is some precedent in microbial systems (1). which involves protein-protein interaction between subunits in a multimeric enzyme. In order 10 
evaluate this possibility, one must consider what is known about the structure of phenylalanine hydroxylase. There is evidence that rat liver phenylalanine hydroxylase is a multimeric protein composed of two electrophoretically distinguishable subunits (molecular weight between 51,000 and 55,000$)(12)$. In addition to the monomers, the enzyme exists as dimers (mol wt 110,000) and tetramers (mol wt 210,000 ) (12). It is not known with certainty, however, which form is catalytically active. Nonetheless, the fact that the enzyme shows cooperative kinetic behavior (in the presence of tetrahydrobiopterin) (12) indicates strongly that a polymeric form of the enzyme is active. It is also known that limited proteolysis of the enzyme leads to a highly active form that can exist only as monomers and dimers (3). Since, as has already been mentioned, there is kinetic evidence that a polymeric form of the enzyme is active, this last finding indicates that the dimer is catalytically active.

With these considerations in mind, and on the assumption that the structure of the human liver enzyme is essentially the same as the rat liver enzyme, the interallelic complementation model would lead to the following picture for the phenotype of patients and their parents. According to this model, the normal hydroxylase is a dimer composed of two different subunits, $\alpha$ and $\beta$, each subunit under the genetic control of an A locus and a B locus. The product of the mutant hyperphenylalaninemia gene would be a modified hydroxylase subunit, $\alpha^{\prime}$ or $\beta^{\prime}$. The hydroxylase in homozygous patients would then have the structure $\alpha^{\prime} \beta$ or $\alpha \beta^{\prime}$ (genotype $\mathrm{A}^{\prime} \mathrm{A}^{\prime} \mathrm{BB}$ or $\mathrm{AAB}^{\prime} \mathrm{B}^{\prime}$ ): these mutant dimers would have $5 \%$ of the normal hydroxylase activity. If the parents are simple heterozygotes (genotype $\mathrm{A}^{\prime} \mathrm{ABB}$ or $\mathrm{AAB} \mathrm{B}^{\prime} \mathrm{B}$ ), their tissues would have equal amounts of two types of enzyme molecules: $\alpha \beta$ and $\alpha^{\prime} \beta$ for the first genotype, and $\alpha \beta$ and $\alpha \beta^{\prime}$ for the second, and their hydroxylase activity would be expected to be about $50 \%$ of normal. To explain the present finding that parents of hyperphenylalaninemic patients have an average of $10 \%$ of the normal activity, one must assume nonrandom combination of the subunits (leading to selective formation of $\alpha^{\prime} \beta$ or $\alpha \beta^{\prime}$ mutant dimers) or more rapid synthesis (or slower degradation) of the mutant subunit compared with that of the normal subunits; either situation could lead to a preponderance of mutant dimers over the normal, fully active dimers.

These arguments are based, in part, on our finding (as mentioned previously), that phenylalanine hydroxylase is composed of nonidentical subunits. It is possible that the enzyme is a multimer of identical subunits, the observed physical differences being caused by partial proteolysis of some of them during the isolation procedure. In this case, the analogous model of negative intraallelic complementation would lead to the same conclusions for the hydroxylase activity in heterozygotes.

If the active form of the hydroxylase were the tetramer $\left(\alpha_{2} \beta_{2}\right)$. the same line of reasoning would lead to the prediction that the parents would have a minimum of about $29 \%$ of the normal hydroxylase activity (i.e., for the case in which the $\alpha$ subunit is the modified one, their tissues would contain three types of enzyme molecules, $\alpha_{2} \beta_{2}, \alpha \alpha^{\prime} \beta_{2}$, and $\alpha^{\prime}{ }_{2} \beta_{2}$, constituting. respectively, 25 , 50 , and $25 \%$ of the hydroxylase population. If the presence of even a single mutant subunit, $\alpha^{\prime}$, in the tetramer led to an enzyme with only $5 \%$ of the normal catlaytic activity, three-fourths of the molecules would have $5 \%$ and one-fourth would have $100 \%$ of the hydroxylase activity, giving an average of $29 \%$ of the normal activity for this population of enzyme molecules).

Although we have not measured the hepatic phenylalanine hydroxylase levels in heterozygotes for classic PKU (i.e.. parents of patients with PKU), it seems likely that they, too, will be found to have less than $50 \%$ of the normal activity. The basis for this prediction is the observation that the ratio of plasma phenylalanine to tyrosine, a reflection of in vivo phenylallanine hydroxylase activity, is similarly elevated for parents of classic PKU patients and for parents of hyperphenylalaninemia patients (9). However, even though PKU homozygotes have a far greater deficit in phenylalanine hydroxylase activity $(0.27 \%$ of normal activity (5)) than do hyperphenylalaninemia patients, it should be evident from the models that have been discussed above that there need be no strict correlation between hydroxylase activity in homozygotes and in heterozygotes; i.e., classic PKU heterozygotes could well have higher hydroxylase activity than hyperphenylalaninemia heterozygotes.

A rigorous test of the validity of the foregoing conclusions must await further studies on the structure of the normal and the mutant enzymes and the availability of additional biopsy samples.

\section{SUMMARY}

Patients with hyperphenylalaninemia have about 5\% of the normal activity of hepatic phenylalanine hydroxylase, whereas their parents, presumed heterozygotes, have an average of $10 \%$ of the normal activity. These results can be explained by the multimeric structure of the hydroxylase.

\section{REFERENCES AND NOTES}

1. Fincham, J. R. S.: Genetic Complementation (W. A. Benjamin, Inc., New York, 1966).

2 Finkelstein, J. D.. Mudd, H. S., Irreverre, F.. and Laster, L.: Homocystinuria due to cystathionine synthase deficiency: The mode of inheritance. Science, 146: 785 (1964).

3. Fisher. D. B., and Kaufman, S.: The stimulation of rat liver phenylalanine hydroxylase by lysolecithin and $\alpha$-chymotrypsin. J. Biol. Chem.. 248: 4345 (1973).

4. Friedman, P. A., Kaufman, S., and Kang. E. S.: Nature of the molecular defect in phenylketonuria and hyperphenylalaninemia. Nature. 240: 157 (1972).

5. Friedman. P. A.. Fisher, D. B., Kang, E. S., and Kaufman, S.: Detection of hepatic phenylalanine 4-hydroxylase in classical phenylketonuria. Proc. Nat. Acad. Sci. U. S. A., 70: $552 \cdot 556$ (1973).

6. Gaull, G. E., and Sturman, J. A.: Vitamin $B_{6}$ dependency on homocystinuria. Brit. Med. J., 3: 532 (1971).

7. Harris, H.: The Principles of Human Biochemical Genetics, p. 173 (American Elsevier, New York, 1970)

8. Hsia, D. Y.: Phenylketonuria and its variants. In: $\Lambda$. G. Steinberg and A. G. Bearn: Progress in Medical Genetics. Vol. V11, p. 29 (Grune \& Stratton. Inc. New York).

9. Kang, E. S., Kaufman, S., and Gerald, P. S.: Clinical and biochemical observations of patients with atypical phenylketonuria. Pediatrics, 45:83 (1970).

10. Kaufman, S.: Phenylalanine hydroxylise of human liver: Assay and some properties. Arch. Biochem. Biophys., 134: 249252 (1969).

11. Kaufman. S and Max, E. E. Studies on the phenylalanine hydroxylating system in human liver and their relationship to pathogenesis of PKU and hyperphenylalaninemia. In: H. Bickel, F. P. Hudson, and L. I. Woolf: Phenylketonuria and Some Other Inborn Errors of Amino Acid Metabolism, pp. 1319 (Georg Thieme Verlag, Stuttgart, 1971).

12. Kaufman. S and Fisher. D.: Purification and some physical properties of phenylalanine hydroxylase from rat liver. I. Biol. Chem., 245: 4745 (1970).

13. Kelley. W. N., Levy, R. I., Rosenbloom, F. M.. Henderson. J. F., and Seegmiller, J. E.: Adenine phosphoribosyl transferase deficiency: A previously undescribed genetic defect in man. J. Clin. Invest. 47: 2281 (1968).

14. Laster. Spaeth. G. L.. Mudd, H. S., and Finkelstein. S. D.: Homocystinurii due to cystathionine synthase deficiency. Ann. Intern. Med., 63: 117 (1965).

15. These statements are supported by the following preliminary data collected by one of us (ESK). Families of phenylketonuric and hyperphenylataninemic children were followed after the diagnosis of the index case. Of the births in the phenylketonuric families, all the children were either classic phenylketonuria or phenotypic normal subjects (no persistent hyperphenylalaninemia noted in four separite blood determinations within the first week of life). Of all the births in the hyperphenylalaninemia families, 10 were phenotypic normals and $4(29 \%)$ were hyperphenylataninemics, close to the $25 \%$ expected for a classic recessive trait.

16. We have recently learned $(H$. Bickel and K. Bartholomé, personal communication) that there is no difference in the activity of phenylalanine hydroxylase in human liver samples obtained by needle and open biopsy procedures.

17. Requests for reprints should be addressed to: S. Kaufman, Ph.D., Laboratory of Neurochemistry. National Institute of Mental Health. 9000 Rockville Pike. Bethesda, Md. 20014 (USA).

18. Accepted for publication April 2, 1975 